\title{
The GALEX Extended Mission: Surveying UV Tracers of the Hidden Side of Galaxy Evolution
}

\author{
D. Christopher Martin and the GALEX Science Team \\ Cahill Center for Astronomy and Astrophysics, California Institute of Technology, USA
}

\begin{abstract}
The Galaxy Evolution Explorer (GALEX) continues its surveys of the ultraviolet sky. GALEX surveys have supported the following galaxy evolution investigations: calibrating UV as a star formation rate tracer, using wide and deep surveys to measure star formation history, studying the evolution of dust extinction and metallicity, selecting and analyzing galaxies in transitory states, finding local analogs to Lyman Break Galaxies, probing and time-dating star formation in a wide variety of physical regimes. Our continuing mission is focussed on relating star formation history and galaxy evolution paths to the properties of dark matter halos and their assembly history, and on beginning to relate the evolution of galaxies to that of black holes and the intergalactic medium. GALEX has proven that the UV is an ideal band to find and map star formation in low mass, low density objects, and potentially in primordial gas. With future UV missions it may be possible to map emission from the intergalactic and circum-galactic medium, and make a definitive connection between galaxy evolution and the cooling, accretion, heating, and enrichment of gas in the cosmic web.
\end{abstract}

\section{INTRODUCTION}

Astrophysicists now depend on panchromatic observations from NASA's Hubble, Chandra, Spitzer, GALEX, and WISE missions, along with ground-based facilities, to analyze complex objects and constrain evolving theories. Ultraviolet (UV) radiation is a key component in such studies, as it is produced by the youngest, most massive stars and hence is an excellent way to locate recent star formation. While some UV capabilities are available on other space observatories, GALEX is unique in combining survey grasp, sensitivity, and mission design to obtain large, homogeneous samples in the UV that can be cross-matched with wide and deep surveys at other wavelengths.

GALEX continues to make exciting new discoveries, such as late-epoch star formation in $\mathrm{E}$ and $\mathrm{SO}$ galaxies, star formation in the primordial HI Leo ring and the first UV shock breakout from a core-collapse supernova. Guest Investigators (GIs) are looking for exoplanets, probing the interiors of White Dwarfs, and exploring possible variations in the stellar Initial Mass Function. Mosaiced images of All-Sky-Imaging Survey (AIS) fields are mapping the Galactic cirrus at unprecedented resolution; only by looking at large areas is it possible to identify some large-scale structures.

The GALEX Extended Mission (EM) is designed to address these focused objectives : 1) Extend the UV/SFR calibration to low mass, low metallicity, and transitional galaxies; 2) Explore the life cycle of dust in galaxies; 3) Quantify star formation history and determine its drivers by linking it to halo mass and assembly history, environment, AGN and their evolution, and the Intergalactic medium (IGM); 4) Uncover a local population of extreme star-forming Lyman Break Analogs; 5) Discover and characterize galaxies 
undergoing transformation; 6) Quantify and explain star formation history in the low mass universe; 7) Probe post-main-sequence evolution; 8) Monitor the dynamic UV sky. These objectives, and the large-area surveys required to meet them, are highly complementary to HST/COS, WFC3, and ACS goals and capabilities, and all three instruments will benefit greatly from contemporaneous operation with GALEX.

\section{GALEX EXTENDED MISSION SURVEYS}

GALEX surveys the sky simultaneously in either one (NUV, 1771-2831 $\AA$ ) or two bands

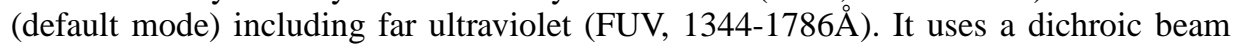
splitter, large-format detectors, and a novel optical design to provide a circular field of view (FOV) of 1.2 degrees. Observations are performed during 25-minute orbital nights (one 1500 second eclipse per 98-minute orbit). In imaging mode, GALEX obtains a resolution of $\sim 5 \operatorname{arcsec}$ FWHM, with $5 \sigma$ sensitivity for point sources of $m_{A B} \sim 23.4$ in 1500 seconds. In grism mode, spectral resolution $\mathrm{R} \sim 100$ is obtained for most objects in the field of view. The grism is rotated to a distinct position angle each orbit to mitigate effects of spectrum overlap. Time-tagged single photon data are stored on-board and telemetered to the ground during three or four daily ground station contacts. The NUV sky is very dark, dominated by diffuse zodiacal light $\sim 27.2 \mathrm{mag} \operatorname{arcsec}^{-2}$ or 30 photon/PSF in one orbit. Astrometric accuracy is $<1 \operatorname{arcsec}(\mathrm{rms})$. A safe NUV count rate limit of $80,000 \mathrm{cps}$ allows us to observe $\sim 26,000 \mathrm{deg}^{2}$ of the sky.

The GALEX EM will yield a twenty-fold increase in survey volume, a factor of ten increase in uniform deep imaging and spectroscopy sky coverage with associated corollary survey data, and a cadenced time-domain survey. The EM survey data support EM Key Science projects, complement targeted GI observations, become available to the community quickly with no proprietary delay, and yield a powerful legacy for present and future researchers.

GALEX LEGACY SURVEY (GLS) [26,000 ORBITS]. With an investment of 26,000 orbits, we will cover the entire AIS footprint at single-orbit integration depth (1500 sec). Because the AIS is photon-limited and the MIS nearly so, this yields an eightfold improvement in limiting flux and a twenty-fold increase in discovery volume over the AIS. The GLS will overlap space-based surveys (WISE, Herschel-ATLAS, Kepler, etc.), ground-based spectroscopic surveys (SDSS I-III, WiggleZ, GAMA, DEEP, VIMOS, zCOSMOS), imaging surveys (PS1, SkyMapper, SDSS, VISTA), and HI surveys (ALFALFA, AGES, ASKAP).

We have entered an age of statistical astrophysics, in which large samples of objects obtained in multi-wavelength surveys are being deployed to answer fundamental questions about the nature of stars, galaxies, and the cosmos, and to probe the evolutionary relationship between different astrophysical objects. The driving themes motivating the EM surveys and the survey sizes are itemized below.

(1) SFR and key physical parameters: GALEX is exquisitely sensitive to star formation over a broad dynamic range of galaxy mass and specific star formation rate. GALEX and optical surveys (e.g., SDSS) give extinction, rough metallicity, and the rate of change of SFR. GALEX probes hot post-main sequence stars even when paired with cool stars. 
(2) Dynamic range: Physical inference comes from comparing physical parameters in objects with widely varying properties, such as galaxy mass. This requires effectively volume-limited surveys to collect comparison samples at the same cosmic epoch and environmental conditions. A major goal of the GALEX EM is to compare the properties of well-studied high mass galaxies to the poorly understood low mass universe that continues to evolve today and provides important clues to the early stages of galaxy formation and evolution. The GALEX EM probes a dynamic range of $10^{4}$ in galaxy stellar mass and $10^{6}$ in SFR.

(3) Rare detections: The UV provides unparalleled insight into transitory evolutionary states and local analogs to early universe objects. Only with the GLS and DLS will sufficient numbers of Lyman Break Galaxy analogs, $\left(\sim 1 \mathrm{deg}^{-2}\right)$, transitional galaxies (evolving from the blue to the red sequence, $\sim 2 \mathrm{deg}^{-2}$ ), red sequence galaxies with no residual star formation, and low mass/metallicity galaxies in all evolutionary states be detected.

(4) Large number power: Only with large samples can objects be subdivided by observed and inferred parameters to elucidate the causes and effects of astrophysical processes driving evolution. An example is the measurement of the star formation "quenching rate" vs. galaxy mass and AGN luminosity. With large samples it is possible to perform unique and insightful statistical analyses, such as stacking to find faint halos of extragalactic gas emission, galaxy-galaxy lensing signals, weak reprocessed Far IR light in low mass objects, weak residual star formation in red, "dead" galaxies, or UVbright QSOs observable by UV spectroscopic missions. Only with large samples can the effects of cosmic variance be overcome.

(5) Complete UV census: The legacy value of the GLS is that it provides a complete survey of the GALEX-available sky. When local GRB or SNe or gravitational-wave hosts are sought, the GLS will provide a SFR in 2/3 of the cases. When WISE or LSST objects are being pre-classified to select for detailed follow-up, the GLS will provide highly-leveraged selection criteria. The GLS will provide a near-complete map of the local 100-300 Mpc low mass universe and the moderate to high galactic latitude Milky Way post-main sequence population and dust distribution.

(6) Faint UV emission: Many objects, structures, and nebulae are intrinsically faint (e.g., low mass, low density, low surface bright-ness galaxies, extended UV emission) and require larger survey volumes and deeper exposures to be identified and imaged.

DEEP LEGACY SURVEY (DLS) [500 ORBITS/YEAR]. To complement the GLS we will obtain deep images (15 ksec/year) over $50 \mathrm{deg}^{2}$ of the SDSS, Pan-STARRS-1, DEEP, and other major space- and ground-based deep photometric and spectroscopic surveys. The resulting deep images will permit a definitive comparison of star-forming galaxies at $z \sim 1$ to $z \sim 0$, a sensitive study of the UV upturn and residual star formation in elliptical galaxies, star formation and chemical evolution of very low mass galaxies, and the properties of extremely red (extincted) star forming galaxies. The DLS is complete at $m_{A B} \sim 25.5$ (confusion-limited depth) at the end of two years.

SPECTROSCOPIC LEGACY SURVEY (SLS) [250 ORBITS/YR]. We will make homogeneous spectroscopic observations in fields that already have publicly available optical spectroscopy (SDSS, DEEP, COSMOS, etc.), extending the coverage to $25 \mathrm{deg}^{2}$ (factor of 5 increase in SDSS and WISE overlap). We will be able to perform multi- and extended-object spectral extraction. 
GALEX is performing a TIME DOMAIN SURVEY (TDS) piggybacked on the DLS. The TDS uses no additional time and has no impact on the prioritization of the EM Surveys. The GALEX TDS exploits the excellent GALEX UV sensitivity, dark UV sky, wide field-of-view, flexibility afforded by photon-counting detectors, repeated visits, and the contrast sensitivity of the UV emission from most variable phenomena. The TDS will complement ground-based TDS (Pan-STARRS-1 [PS1], PTF).

GUEST-INVESTIGATOR (GI) PROGRAM. The GALEX Extended Mission includes a vigorous GI Program that uses roughly $25 \%$ of the observation time, and supports $\sim 30$ investigations per year.

\section{SFR vs. UV}

GALEX identifies star formation over an astonishing range of scales, modalities, and environments because UV luminosity scales with the number of $\mathrm{O}, \mathrm{B}$, and A stars in a galaxy and thus measures the star formation rate (SFR) averaged over $\sim 10^{7}-10^{8}$ years. This timescale corresponds to driving dynamical timescales (e.g., spiral density waves, tidal interactions, starburst region infall, and gas starvation in clusters). Although dust extinction absorbs some UV and reradiates it in the mid- and far-infrared, the UV is still a sensitive tracer of SFR: with typical UV extinctions $\left(\mathrm{A}_{U V} \sim 1.3\right)$, a SFR $\sim 1 \mathrm{M}_{\odot} y r^{-1}$ can be measured at $z \sim 1$ in $30 \mathrm{ksec}$. GALEX surveys, augmented by multiwavelength data, provide a definitive calibration of the UV-SFR relationship in various galaxy classes, showing for example that $\mathrm{H} \alpha$ - and UV-derived SFRs correlate extremely well for galaxies with a systematic NUV-r color dependence [1], and UV-derived SFRs are far more reliable than $\mathrm{H} \alpha$ in cases with weak SF superimposed on passively evolving components [2]. Thus UV is ideal for studying the complex evolutionary phenomena that link the star-forming (blue) sequence to the passively evolving (red) sequence. This calibration will be crucial for physical interpretation of the rest UV emission from young (high redshift) galaxies observed with HST and JWST.

The UV-Optical color-magnitude diagram (UVOCMD), a powerful tool for separating and relating galaxy types, properties, and evolutionary histories, can be considered an HR diagram for galaxies. This results from the extraordinary leverage obtained with the UV (SFR) - optical/NIR (stellar mass) color. We have shown in a very general way that the dust-corrected UV-Optical/NIR color is a precise measure of the specific SFR (SSFR) over a remarkable dynamic range: from starbursts processing their gas in $10^{8}$ years to dying galaxies exhausting their last shreds of star-forming gas or rejuvenated by tiny new accretions of intergalactic material. The UVOCMD, which can be measured in very distant samples, delineates galaxies by hard-to-measure spectroscopic properties such as $\mathrm{D}_{n}(4000)$ and $\mathrm{H}_{\delta A}$ (stellar age metrics), stellar mass and mass-to-light ratio $(\mathrm{M} / \mathrm{L})$, metallicity, morphological parameters, and the properties of their active nuclei [3, 4]. These UV-H $\alpha$, MIR, FIR, and spectral index relationships reveal that the blue sequence (active star forming galaxies) at $z \sim 0$ is a regular sequence in which a galaxy's stellar mass is the major predictor of galaxy properties 


\section{THE LIFE CYCLE OF DUST AND STAR FORMATION HISTORY}

Dust typically reprocesses half the UV/optical light into the infrared in star forming regions. Our ability to determine galaxies' physical properties is largely delimited by our ability to correct for dust effects. We are developing a definitive method for extinction correction and tying that to star formation history. We are doing this by determining dust properties, and resulting extinction laws, in locations ranging from the zodiacal belt to distant galaxies.

Extinction and Galaxy Physical Parameters (GPP) across the UVOCMD. Several pioneering studies $[1,2,5,6,7,8,9]$ have been synthesized into a single method for measuring extinction with a precision of $\sigma_{A(U V)}<0.4 \mathrm{mag}$ [10]. The method works for galaxies spanning the UVOCMD, at all redshifts, even when the extinction law exhibits unpredictable variations: $\mathrm{D}_{n}(4000)$ determines an extinction-independent age, and the exquisitely sensitive UV-optical color gives both age and extinction.

Extinction and Star Formation History (SFH). Extinction laws in other galaxies are poorly known, but are key in extracting physical parameters from galaxy spectral energy distributions (SEDs). Most high-z galaxy SED work relies on the Calzetti law [11] based on a handful of local starburst galaxies and IUE spectra. The connection of these laws to Milky Way line-of-sight laws is complicated by, e.g., scattering, geometry, metallicity, and grain sizes.

Crucial questions are: Is the starburst extinction law universal, or in need of revision? How does the extinction law vary with SSFR and mass? Does the extinction law vary across or within a galaxy? Does the Galaxy's $2175 \AA$ bump ever appear in other galaxies; if so, is it correlated with Polycyclic Aromatic Hydrocarbon (PAH) emission? GALEX is already addressing these questions. Spitzer and GALEX data show tight calibrations, e.g., relating UV-optical color or spectroscopic indices such as $\mathrm{D}_{n}(4000)$ to the IR eXcess $\left(I R X=L_{F I R} / L_{U V}\right)$, stellar age, total SFR, and PAH-derived SFR in galaxies. At $z \sim 1$ the IRX-UV spectral slope relation for all galaxies is consistent with that for local starbursts, but not with local normal galaxies. The extraordinary range of IRX within/between galaxies traces gas surface density (star formation efficiency), and gas metallicity. Because IRX correlates tightly with metallicity, it measures the evolutionary age of a galaxy: galaxies with high SFRs quickly become metal rich and show high extinction, while young galaxies exhibit low IRX and are UV-bright. GALEX UV selection yields a uniquely wide range of stellar mass, metallicity, and age. [12]. The GLS and DLS will be combined with Herschel/ATLAS and other major surveys to measure IRX using the full Far IR SED over several $100 \mathrm{deg}^{2}$. The GLS will be combined with WISE $22 \mu \mathrm{m}$ (a proxy for bolometric FIR luminosity) to measure IRX in $10^{7}$ galaxies; extinction laws for each galaxy type will be determined, including the rare low mass, low SSFR, and Lyman-break-analog galaxies.

Milky Way extinction can be measured using the classical approach of deviations in the number count distributions of extragalactic sources. Pilot programs show this program can obtain an extinction precision of $\sigma\left[A_{N U V} / E_{B-V}\right] \sim 0.06$ on 20 arcmin scales [13]. With GLS coverage, we will be able to link dust extinction with scattering from cirrus and diffuse dust. Stacked galaxy and stellar spectra from the SLS will show the relation of any detected $2175 \AA$ bump to the local cirrus and PAH density.

Milky Way Cirrus traces dust in the diffuse ISM, and is mapped beautifully by 
GALEX. FUV cirrus is often dominated by $\mathrm{H}_{2}$ fluorescence $(\mathrm{H} 2 \mathrm{~F})$, while NUV cirrus is pure scattering of the Interstellar Radiation Field (ISRF). UV emission and extinction, combined with FIR and HI, constrains dust properties (e.g., phase function, albedo, grain sizes) and dust-to-gas ratios. GLS depth and sky coverage is required to map cirrus to the lowest column densities and to delineate its role in a full radiative model of the ISRF. Detailed comparison of GALEX EM dust scattering maps with WISE's PAH maps will reveal how the PAH life cycle relates to the dust.

\section{GALAXY PHYSICAL PROPERTIES AFFECTING STAR FORMATION HISTORY}

The sensitivity of the GALEX UV to low levels of SF will constrain the mode of gas accretion (cold vs. hot) and those physical processes responsible for regulating star formation: feedback, cooling, stripping, strangulation, harassment, nuclear activity, and others. To address these questions in the present day Universe and as a function of look-back time, the GLS is essential. The MIS Survey depth, when matched to SDSS, is required to identify transition and red sequence galaxies, and to sample adequately the full mass range of galaxies. The leverage gained by matching the GLS with other datasets (e.g. WISE, Herschel, Pan-STARRS) will develop a complete picture of star formation to enable these investigations.

Only with large, homogeneous galaxy samples can we explore the widest possible dynamic range in GPP. A viable predictive theory of baryonic structure formation requires detailed tests of semi-analytic models (SAMs) using measurements of star formation history (SFH) vs. other factors such as gas density and phase, galaxy type, nuclear activity (AGN), galaxy clustering, environment, dark matter halo properties, or assembly history.

The dependence of SFH on galaxy stellar mass determines the evolution of galaxies over the range $10^{9}<M_{*} / M_{\odot}<10^{12}$, straddling the "transition mass" of $M_{*}=10^{10.5} M_{\odot}$, the mass break between red and blue galaxies [12]. The GLS dataset, when matched to the new Pan-STARRS photometry, permits measurement of the recent SFH of the Universe as a function of redshift and stellar mass. These will critically place the most recent 5 Gyr in the context of the cosmic SFH since $\mathrm{z}=7$, being defined now with increasing precision.

The number density, $\phi\left(M_{*}, S S F R, z\right)$ measures the distribution of galaxies in the UVOCMD $\left(M_{*}\right.$, SSFR) plane. We will determine how this fundamental distribution changes with redshift and its relationship to other individual galaxy physical properties such as morphology, nuclear properties, size, extinction, metallicity and age. GLS samples are required to robustly measure low number density regimes that uniquely test models.

Environment can be measured directly via, e.g: galaxy number density, membership and location in groups/clusters, dominance in groups, location in the Cosmic Web structure, or indirectly via clustering. We will connect galaxy environment with SFH as already demonstrated in smaller samples $[14,15,16]$. Galaxy clustering (and bias) is a strong function of current SSFR, indicating that a significant fraction of passive and transitioning (green valley) galaxies are satellites inside the dark matter haloes of 
groups/clusters [16]. Measuring environment directly in sparsely populated regions of the UVOCMD, representing galaxies in rare evolutionary states, with fine sampling in stellar mass and SSFR, will require the large sample provided by the GLS.

SAMs connect the growth of dark matter structures to SFH/galaxy evolution. The strongest constraints relate DMH properties to the (observable) SFH. Our goal is to determine the distribution of DMH mass vs. $\mathrm{M}_{*}$ and SSFR simultaneously over a wide range of both with reasonable accuracy, which is not feasible without the GLS/SDSS.

Weak lensing analysis constrains the typical DMH mass of central galaxies and the fraction of galaxies that are satellites. Clustering can also constrain the distribution of DMH masses, but this requires a large contiguous area $\left(\sim 10,000 \mathrm{deg}^{2}\right.$, MIS depth $)$, which the GLS will provide. Assuming transition galaxies occupy forming DMHs, comparing these two approaches using the 4 year GALEX/SDSS will test nonlinear structure formation (e.g., the assembly bias ) at the $2 \%$ level.

The distribution of physical and environmental properties across the UVOCMD will complement the suite of mostly optical data currently used to confront and constrain SAMs. In doing so, they will challenge current models and motivate refined theoretical treatments of galaxy evolution. The new GALEX-based constraints will be especially powerful because the UV is sensitive to the time scales on which many processes are believed to operate.

GALEX examines the only period in the history of the Universe where the cosmic SFR is declining $(0<z<1)[12,17,18]$. By providing an exquisitely sensitive measurement of SF to very low levels, GALEX offers the only way to explore the relationship of gas to halos (by proxy to star formation) over large angular areas, over half a Hubble time, thereby providing unique insights into the physics of galaxy evolution.

\section{LYMAN BREAK ANALOGS}

GALEX has enabled an exciting discovery: a rare population of low-redshift starbursts with properties remarkably similar to high-redshift Lyman Break Galaxies (LBGs) that we are calling Lyman Break Analogs (LBAs) [19, 20, 21]. These galaxies, selected based on their extremely high UV luminosity and surface brightness, offer a unique local probe into the physics of unobscured, intensely star-forming regions. The initial sample of 50-100 such objects has been studied using HST, Spitzer, ground-based Integral Field Units (IFUs, Keck/VLT) and at $\mathrm{mm}$ /radio wavelengths, with observations providing new insights into intense star formation at all redshifts, especially relevant to the newly discovered, UV-selected objects at $z \sim 7-8$. Interpretation of this sample has been limited by sample size; there is tremendous value in selecting more low redshift examples for detailed follow-up. For example, only a restricted subset is sufficiently luminous and compact to enable follow-up with COS/HST. Six LBAs contain extremely massive compact star forming clumps or dominant central objects (DCOs), and we anticipate identifying a set of 500 DCOs over the course of the mission. Another subset of $\sim 30$ is ideally suited for high resolution HST follow-up with ACS, allowing a study of faint optical light/fine structures which provide important clues into the recent dynamical/merger history of these galaxies [21, 22]. The Extended Mission (GLS) will produce large $(\sim 20,000)$, homogeneous samples of rare UV luminous galaxies and will 
obtain an unprecedented set of $\sim 300$ at low redshift for detailed study.

GALEX deep surveys show that LBAs are the fastest evolving component of the UV galaxy population and deviate from the $z \sim 0$ blue sequence. They are less dustattenuated than other local starbursts such as ULIRGs, consistent with an early stage of chemical evolution. HST images of GALEX-discovered LBAs reveal that they represent a complex merger of multiple, lower mass, gas rich subunits that echo the morphology and physical properties of high-z LBGs. These images also reveal that their basic structure and origin may be hidden at high-z but is revealed at low-z.

We are characterizing LBAs and their environments, to investigate how the high SFRs are triggered and/or sustained, and to use the compact systems as local laboratories for detailed investigation of the processes in LBGs. Hierarchical models predict multiple merging and accretion events, which are likely to induce starbursts on top of a smooth SF history. UV, $\mathrm{H} \alpha$, and MIR/FIR SFR metrics sample different timescales and are thus sensitive to starburst history. Our measurement of the fraction, duration and amplitude of SF occurring in bursts vs. mass, and the relationship between extinction and starburst age will provide a powerful new test of hierarchical models. GALEX UV observations are particularly sensitive to starbursts in less evolved, high gas-fraction, lower metallicity accreting sub-units, which are likely to play a fundamental role in galaxy assembly. GLS observations yield the required high-quality photometry and GPPs for LBAs and their neighborhoods.

A unique and unprecedented aspect of this key project is the ground-based IFU follow-up. LBGs and other massive star-forming galaxies at high redshift are now being studied using NIR integral field spectrographs, often with Adaptive Optics, where the $\mathrm{H} \alpha$ line is being used to study the kinematics and resolve star formation in these extreme systems (on 500-1000 pc scales). While these early studies suggest a great diversity of kinematical types, a substantial number of velocity field maps suggest disk-like rotation, but with unusually high velocity dispersion. In an analogous manner, our local samples of LBAs can be studied on 100 pc scales using NIR/IFU observations of $\mathrm{P} \alpha$ [23], an emission line now established as a "ground-truth" star formation rate indicator because it is relatively unattenuated. These similar and powerful new data sets are providing a unique tool to compare the dynamics of galaxies across redshift.

\section{TRANSITION GALAXIES - WATCHING GALAXY EVOLUTION}

Because of the remarkable sensitivity of the UVOCMD to low levels of star formation, GALEX has dramatically changed the study of galaxies at the end of their star-forming phase and transitioning between the blue and red sequences. This is crucial for developing a theory of baryonic structure formation, since the processes that control the onset and end of star formation are the most poorly understood in the field of galaxy evolution.

In 2007 we showed that the galaxy distribution is not simply bimodal, that there is a major population of "transitional galaxies" in what we termed the "green valley" between the actively star-forming blue sequence and the passively evolving red sequence [4]. We developed a method to measure the mass flux of galaxies from the blue to the red sequences using the UVOCMD and SDSS spectral or morphological indices [3, 24]. We found that local intermediate-color galaxies are evolving off the blue sequence and 
causing the fall in the cosmic SFR and mass growth of the red sequence. We also determined the rate at which SF is quenched in these rare transitional objects, and tried to relate this to the remarkable abundance of AGN in the green valley. Because models had a very simplified mechanism for halting star formation they did a poor job of reproducing the green valley population. At the same time, we realized that galaxies are moving in two directions in the green valley. Quenching galaxies move toward the red sequence; passively evolving galaxies, undergoing new bursts of star formation from accretion or mergers, move from red to blue in a way that may be moderated by an AGN.

Recognizing this two-way flow across the green valley, we have developed a new approach using UV and optical data to measure the Star Formation Acceleration (SFA), the rate of change of the (NUV-i) color. SFA is positive for quenching galaxies, and negative for galaxies undergoing starbursts. Preliminary work uses a subset of the 500 $\mathrm{deg}^{2}$ SDSS/MIS sample to derive SFA vs. NUV-i color in two mass bins, above and below the "transition mass" of $10^{10.5} M_{\odot}$. Lower mass galaxies in the green valley are mostly quenching, while higher mass galaxies are both quenching and bursting. This could mean that lower mass galaxies are accreting and becoming satellite galaxies, having their star forming gas tidally and/or ram-pressure stripped, while higher mass galaxies are receiving this gas and reacting with new star formation. These mass differences are extremely important for galaxy models, and obtaining significant numbers of low mass green-valley galaxies and comparing them to high mass galaxies requires the GLS.

In the next two years we will "calibrate" SFA by measuring it in nearby galaxies where physical processes are clearly triggering bursts (e.g., by gas compression, tidal interactions, merging, accretion) or quenching star formation (e.g., by ram pressure stripping, harassment, or tidal stripping). We can correlate SFA over the UVOCMD with other galaxy physical and environmental parameters, testing models for galaxies in transition and the role of AGN feedback. By combining this quantitative transition metric with the environmental and halo measurement techniques discussed above, we can with the GLS perform a sensitive test of a halo-merger driven star formation history. Finally, by self-consistently combining SFA and the bivariate UVOCMD luminosity function, and measuring this as a function of redshift with the DLS, we can construct a picture of the complex flow of galaxies over this "HR-diagram" and perhaps as directly as possible watch the evolution of galaxies from the epoch of star formation to today.

\section{THE LOW-DENSITY UNIVERSE}

One of the seminal contributions of the GALEX mission has been the exploration of SF in low-density environments. GALEX is uniquely suited to probe this environment due to its large field-of-view and sensitivity while operating in the low background space UV. Quantifying SF in this environment with $\mathrm{H} \alpha$ or FIR is challenging because SFRs are low and the dust content is negligible.

Extended UV (XUV) Disks. GALEX has revolutionized the understanding of star formation in outer, optically-faint portions of low-redshift galaxies. XUV disks have UV-bright regions of SF located at extreme radii reaching many times the optical extent of the galaxy [25]. XUV disks are late-type galaxies still actively building their stellar disk in the outer, low-density environment. Prior to GALEX, these regions were consid- 
ered to be stable against SF as determined by the dynamics and density of the gas. The UV perspective revealed that such activity is far more common than previously realized ( $\sim 30 \%$ of late-type galaxies ) on the basis of deep $\mathrm{H} \alpha$ imaging. Important implications include chemical enrichment, dust production, and turbulent support of extended gaseous reservoirs. In a broader view, galaxies with XUV-disks represent a population of objects undergoing inside-out structural metamorphosis, whether fueled via gas accretion from the IGM and galaxy mergers or the slow processing of long-lived gas disks. The physical nature of the XUV disks and their role in the evolution of galaxies remains uncertain. Further progress requires both detailed studies of individual objects as well as large statistical samples, which the GLS will provide.

SF Efficiency in Low Surface Brightness (LSB) Galaxies. UV based SFRs have shown that LSB galaxies lie below the extrapolation of the standard SF law [26]. A similar downturn has been detected in the outer low-density regions of normal spiral galaxies as well $[25,27]$. This downturn is consistent with some theoretical models that predict lower star formation efficiencies in LSB galaxies due to the declining molecular fraction with decreasing density.

IMF Variations Suggested by Low Mass/Dwarf Galaxies. Two GI Legacy programs have demonstrated that as one moves to low mass systems or low surface brightness systems or simply low SFR systems, the H $\alpha /$ UV SFR ratio declines dramatically $[28,29]$. Why is hotly debated. An intriguing possibility is that either the IMF is nonuniversal (top-light) in the low-density regime, or the effective galaxy-wide IMF has a variable (steeper) upper end that correlates with SFR. Regardless of why, some of the consequences are clear: non-UV based tracers can underestimate the SFR in low density regimes by up to an order of magnitude and if the IMF is indeed non-universal then much of what is assumed about galaxy evolution must be rethought.

Dwarf Galaxy Formation in Primordial Context. GALEX detects low-mass lowSFR systems with ease. The discovery of a new class of dwarf galaxies forming out of the massive Leo Ring is a prime example [30]. This 200-kpc-wide structure orbiting the Leo I group is a candidate primordial cloud. GALEX has found dozens of UV clumps coincident with HI peaks within the ring suggesting the recent onset of SF with low metallicity $(Z \sim 1 / 50-1 / 5)$. HI studies indicate the ring structure lacks dark matter. If structures like these were common in the early Universe, they may have produced a large, yet undetected population of faint, metal-poor, halo-lacking dwarf galaxies.

Low Density Hosts of Extreme Supernovae. Wide field synoptic surveys are finding that GRBs and the most luminous SNe prefer low-mass dwarf galaxies. The first confirmed electron-positron pair instability supernova (SN2007bi) was hosted by a nearby dwarf galaxy having an absolute magnitude of $\mathrm{M}_{r}=-16.3$ and metallicity of $12+\log [\mathrm{O} / \mathrm{H}]=8.25$. This low metallicity and the high initial mass required by the pairinstability models $\left(\sim 200 \mathrm{M}_{\odot}\right)$ combined with the fact that extremely luminous supernovae are very rare in larger mass galaxies implies that the star formation conditions in nearby dwarf galaxies are different. These luminous $\mathrm{SNe}$ trace these unusual conditions and place direct constraints on the upper end of the initial mass function [31].

In the next two years the GLS will provide significant progress toward a VolumeLimited Survey to $M_{U V}=-12$ to $100 \mathrm{Mpc}$ and $M_{U V}=-14$ to $300 \mathrm{Mpc}$. Since low mass galaxies exhibit low extinction, this corresponds to a SFR limit of 0.001 and $0.02 M_{\odot} / y r$ over volumes of $3 \times 10^{6}$ and $7 \times 10^{7} \mathrm{Mpc}^{3}$ netting an estimated $10^{5}$ and $10^{6}$ galaxies. The 
surface brightness limit of $\mu_{A B}=28$ corresponds to a SFR/area of $10^{-4} M_{\odot} y r^{-1} k p c^{-2}$, a factor of $\sim 30$ below the canonical star formation threshold and corresponding to a gas surface density of $\sim 1 \mathrm{M}_{\odot} p c^{-2}$.

Low mass galaxies are truly galaxies in the process of forming, and we still do not understand the physics behind this long delay. Star forming galaxies in the local 100-300 Mpc volume are likely to be the hosts for GRBs, high-luminosity SNe, and the first gravitational wave sources detected by Advanced LIGO and LISA, and for a variety of transients discovered by PTF, PS1, and LSST. A volume-limited survey of the low mass universe will provide a definitive sample of the lowest mass star-forming systems accessible to detailed study in the next few decades. It will generate a sample large enough to test various theories for the pronounced drop in $\mathrm{H} \alpha / \mathrm{UV}$ ratios for lowmass, low-density regimes, including statistical models of the IMF and the impact of SF history. It will sample a wide range of cosmic environments and densities, including filaments and voids, permitting a test of scenarios for the impact of the cosmological context on fueling, feedback, and SF inhibition. It will provide a definitive survey for unknown star forming regions such as that discovered in the Leo Ring, and an excellent complement to SKA-precursor HI surveys such as ASKAP. Finally, it will allow imaging the entire ALFALFA survey footprint, netting a sample of $>10^{4}$ nearby star-forming galaxies with both gas masses and redshifts with which theories for the formation and regulation of XUV disks could be tested.

\section{ACKNOWLEDGMENTS}

GALEX is a NASA Small Explorer, launched in April 2003. We gratefully acknowledge NASA's support for construction, operation, and science analysis for the GALEX mission, developed in cooperation with the Centre National d'Etudes Spatiales of France and the Korean Ministry of Science and Technology.

\section{REFERENCES}

1. Treyer, M., Schiminovich, D., Johnson, B., et al., ApJS 173, 256 (2007)

2. Salim, S., Rich, R. M., Charlot, S., et al., ApJS 173, 267 (2007)

3. Martin, D. C., Wyder, T. K., Schiminovich, D., et al., ApJS 173, 342 (2007)

4. Wyder, T. K., Martin, D. C., Schiminovich, D., et al., ApJS 173, 293 (2007)

5. Seibert, M., ApJL 619, L55 (2005)

6. Salim, S., ApJL 619, L39 (2005)

7. Buat, V., ApJL 619, L51 (2005)

8. Johnson, B. D., Schiminovich, D., Seibert, M., et al., ApJL 644, L109 (2006)

9. Johnson, B. D., Schiminovich, D., Seibert, M., et al., ApJS 173, 392 (2007)

10. Martin, D. C., et al., in preparation (2010)

11. Calzetti, D., Kinney, A. L., Storchi-Bergman, T., ApJ 429, 582 (1994)

12. Martin, D. C., Small, T., Schiminovich, D., et al., ApJS 173, 415 (2007)

13. Drinkwater, M., WiggleZ Team, GALEX Team, in preparation (2010)

14. Milliard, B., ApJS 173, 503 (2007)

15. Heinis, S., Milliard, B., Arnouts, S., et al., ApJS 173, 503 (2007)

16. Heinis, S., Budav Ãąri, T., Szalay, A. S., et al., ApJ 698, 1838 (2009)

17. Arnouts, S., Schiminovich, D., Ilbert, O., et al., ApJL 619, L43 (2005)

18. Schiminovich, D., ApJL 619, L47 (2005) 
19. Heckman, T., ApJL 619, L35 (2005)

20. Hoopes, C. G., Heckman, T. M., Salim, S., et al., ApJS 173, 441 (2007)

21. Overzier, R., ApJ 677, 37 (2008)

22. Overzier, R., et al., submitted (2010)

23. Basu-Zych, A. R., Gon calves, T. S., Overzier, R. ApJL 699, L118 (2009)

24. Schiminovich, D., Wyder, T. K., Martin, D. C., et al., ApJS 173, 315 (2007)

25. Thilker, D., ApJL 619, L79 (2005)

26. Wyder, T. K.; Martin, D. C., Barlow, T. A., et al., ApJ 696, 1834 (2009)

27. Boissier, S., Gil de Paz, A., Boselli, A., et al., ApJS 173, 524 (2007)

28. Lee, J. C., Gil de Paz, A., Tremonti, C., et al.,ApJ 706, 599 (2009)

29. Meurer, G. R., Wong, O. I., Kim, J. H., et al., ApJ 695, 765 (2009)

30. Thilker, D. A.; Donovan, J., Schiminovich, D., et al., Nature 457, 990 (2009)

31. Neill, D., et al., in preparation (2010) 